\title{
Como Evitar a Formação de Substâncias Tóxicas durante a Absorção de Dióxido de Carbono pela Cal Sodada com Uso de Anestésicos Halogenados *
}

\section{Preventing Toxic Substances Production during Carbon Dioxide Absorption by Soda Lime with Halogenate Anesthetics}

\author{
Renato Ângelo Saraiva, TSA ${ }^{1}$
}

\section{RESUMO}

Saraiva RA - Como Evitar a Formação de Substâncias Tóxicas durante a Absorção de Dióxido de Carbono pela Cal Sodada com Uso de Anestésicos Halogenados

JUSTIFICATIVA E OBJETIVOS: A cal sodada desde o início do seu uso sempre apresentou algumas complicações que resultaram em dificuldade na sua aplicabilidade. No entanto, devido as grandes vantagens que oferecia em relação a redução do fluxo de gases frescos, despoluição da sala de cirurgia e umidificação do sistema de inalação e via aérea, fizeram com que continuassem as pesquisas para que pudesse ser melhorada e corrigida de forma que a continuidade da sua utilização fosse assegurada. Atualmente existe o problema da desidratação com elevação da temperatura e da degradação metabólica dos anestésicos halogenados que necessitam de cuidados especiais para evitar a formação de produtos tóxicos. CONTEÚDO: Existe uma reação em cadeia a partir da cal sodada desidratada ou ressecada com baixos volumes percentuais de água. Há aumento da temperatura, maior absorção de anestésico halogenado para o interior do granulo de cal em seguida maior degradação metabólica das moléculas destes agentes e conseqüentemente a produção de substâncias tóxicas como o Composto $A$ pela reação dos hidróxidos com o sevoflurano. Há também formação de monóxido de carbono produzido da mesma forma pela reação entre os halogenados e as bases fortes da cal. O composto Aé nefrotóxico e o monóxido de carbono leva a hipóxia e alterações graves da coagulação do sangue. Além dos cuidados para a hidratação da cal sodada é possível usá-la sem conter as bases fortes como os hidróxidos de potássio e de sódio, contendo apenas hidróxido de cálcio para evitar excessivo aumento da temperatura e grande degradação metabólica dos halogenados sem prejudicar a absorção do dióxido de carbono.

CONCLUSÕES: Deve-se ter o cuidado em usar a cal sodada mais recente possivel e quando ela fica exposta ao meio ambiente (ar seco) por muitas horas como por exemplo em um final de semana (mais de 48 horas) é recomendável colocar água, de preferência destilada, na relação de $25 \mathrm{ml}$ para cada $500 \mathrm{~g}$ de cal. Atualmente a indústria está bem informada sobre

\footnotetext{
${ }^{*}$ Recebido do (Received from) Hospital SARAH - Brasília, DF

1. Coordenador de Anestesiologia da Rede SARAH de Hospitais do Aparelho Locomotor
}

Apresentado (Submitted) em 15 de julho de 2003

Aceito (Accepted) para publicação em 23 de setembro de 2003

Endereço para correspondência (Correspondence to)

Dr. Renato Ângelo Saraiva

Hospital Sarah

SMHS Quadra 501 - Conjunto A

70330-150 Brasilia, DF

(C) Sociedade Brasileira de Anestesiologia, 2004 o problema da composição da cal, então, deve-se preferir a cal sodada que tenha somente o hidróxido de cálcio e seja totalmente desprovida de hidróxido de potássio e hidróxido de sódio.

Unitermos: EQUIPAMENTOS: cal sodada.

\section{SUMMARY}

Saraiva RA - Preventing Toxic Substances Production during Carbon Dioxide Absorption by Soda Lime with Halogenate Anesthetics

BACKGROUND AND OBJECTIVES: Since the beginning, soda lime use has presented some complications which resulted in its difficult application. However major advantages such as decreasing fresh gas flow, anesthetic consumption and operating room pollution, and improving breathing system and airway humidity, have pushed research forward to improve it and assure the continuity of its use. Currently, there are problems with dehydration, increased temperature and metabolic degradation of halogenate agents, which require special care to prevent toxic substances formation.

CONTENTS: There is a chain reaction as from dehydrated or dried out soda lime with very low percent volume of water. There is increased temperature, more halogenate anesthetic absorption by lime granules, followed by higher metabolic degradation of these agents'molecules and, as a consequence, the production of toxic substances, such as Compound A by reaction of hydroxides with sevoflurane. There is also carbon monoxide production by reaction of halogenate anesthetics and strong lime bases. Compound $A$ is nephrotoxic and carbon monoxide may lead to hypoxia and severe coagulation problems. In addition to care with soda lime hydration it is possible to use it without strong bases, such as potassium and sodium hydroxides, with just calcium hydroxide to prevent excessive temperature increase and major metabolic degradation of halogenate anesthetics without impairing carbon dioxide absorption.

CONCLUSIONS: Care should be taken to use the newest possible soda lime; and when it is exposed to environment (dry air) for many hours, such as during weekends (more than 48 hours) it is recommended to add distilled water in the ratio of $25 \mathrm{~mL}$ to $500 \mathrm{~g}$ of soda lime. Industry is currently well aware of lime composition problems so, soda lime containing exclusively calcium hydroxide and totally potassium and sodium hydroxide-free should be preferred

Key Words: EQUIPMENTS: soda lime

\section{INTRODUÇÃO}

A absorção de dióxido de carbono no sistema respiratório do aparelho de anestesia permite que seja utilizado fluxo de gases fresco mais baixo para reduzir o consumo de anestésico, manter a temperatura corporal do paciente, 
conservar a umidade da via aérea e evitar poluição na sala de cirurgia.

Em trabalhos de Wilson ${ }^{1} \mathrm{e}$ Waters ${ }^{2}$ há relatos que o primeiro uso da absorção de dióxido de carbono em anestesia foi em 1906 por um cirurgião alemão que utilizou um filtro do equipamento de salva- vidas de minas subterrâneas e a seguir o farmacologista Dennis Jackson em 1915 desenvolveu um filtro para a cal sodada que era à base de hidróxido de sódio e a absorção do dióxido de carbono resultava em grande aumento da temperatura. Por esta razão Wilson modificou a composição da cal que passou a ser à base de hidróxido de cálcio.

Em 1923, Ralph Waters começou a usar a absorção de dióxido de carbono rotineiramente, em anestesia e publicou os seus resultados em $1926^{3}$. Ele utilizou um filtro com capacidade de $500 \mathrm{ml}$ próximo a boca do paciente. O fluxo de gás fresco era de $0,5 \mathrm{I} \cdot \mathrm{min}^{-1}$ e entrava na parte superior do filtro que se ligava com o tubo traqueal ou máscara facial. Na parte inferior do filtro era colocada uma bolsa respiratória. Este sistema era chamado "to and fro" traduzido como vai e vem.

A composição da cal sodada continua até hoje com a mesma constituição: hidróxido de cálcio 95\%, hidróxido de sódio, $4,5 \%$ e sílica $0,2 \%$ para tornar a cal mais consistente e evitar a formação de pó.

Após esta mistura era adicionada água em volume de $10 \%$ a $22 \%$ (média de 15\%). Sendo depois transformada em grânu$\operatorname{los}^{4}$.

Posteriormente foi idealizada uma outra mistura para a cal sodada que continha hidróxido de potássio $1 \%$ hidróxido sódio $4 \%$, sílica (pequenas quantidades cerca de $0,2 \%$ ), água $14 \%$ a $19 \%$ e o restante para completar $100 \%$ hidróxido de cálcio ${ }^{5}$.

A cal baritada contém $20 \%$ de hidróxido de bário, $1 \%$ de hidróxido de potássio, percentual de água menor do que a cal sodada (cerca de $8 \%$ ), e hidróxido de cálcio para completar $100 \%{ }^{6}$. $\mathrm{Na}$ tentativa de manter os grânulos mais resistentes a tornar-se pó foi introduzida a cal baritada que substitui parte (cerca de 20\%) do hidróxido de cálcio por hidróxido de bário. No entanto esta produz maior aquecimento durante a absorção de dióxido de carbono ${ }^{6}$.

São utilizadas pequenas quantidades de substâncias indicadoras que mudam de cor quando o $\mathrm{pH}$ diminui. O mais usado até hoje é o etil violeta que é incolor e torna-se violeta quando o pH fica abaixo de $10,3^{7}$.

O contato do dióxido de carbono com a cal sodada, produz, as seguintes reações:

Em uma $1^{\text {a }}$ fase o dióxido de carbono $\left(\mathrm{CO}_{2}\right)$ combina com a água $\left(\mathrm{H}_{2} \mathrm{O}\right)$ na cal formando ácido carbônico $\left(\mathrm{H}_{2} \mathrm{CO}_{3}\right)$.

$\mathrm{CO}_{2}{ }^{+} \mathrm{H}_{2} \mathrm{O} \rightarrow \mathrm{H}_{2} \mathrm{CO}_{3}$

Em uma $2^{a}$ fase, o ácido carbônico reage com o hidróxido de sódio $(\mathrm{OH} N a)$ e o hidróxido de cálcio $(\mathrm{OH})_{2}$ Ca produzindo carbonato de sódio $\mathrm{CO}_{3} \mathrm{Na}_{2}$ e carbonato de cálcio $\mathrm{CO}_{3} \mathrm{Ca}$ e liberando água.

$2 \mathrm{NaOH}+2 \mathrm{H}_{2} \mathrm{CO}_{3}+\mathrm{Ca}(\mathrm{OH})_{2} \rightarrow \mathrm{CaCO}_{3}+\mathrm{Na}_{2} \mathrm{CO}_{3}+4 \mathrm{H}_{2} \mathrm{O}$
As reações do dióxido de carbono com a cal baritada se processam como segue ${ }^{8,9}$.

$$
\begin{aligned}
& 9 \mathrm{CO}_{2}+9 \mathrm{H}_{2} \mathrm{O} \rightarrow 9 \mathrm{H}_{2} \mathrm{CO}_{3} \\
& 9 \mathrm{H}_{2} \mathrm{CO}_{3}+9 \mathrm{Ca}(\mathrm{OH})_{2} \rightarrow 9 \mathrm{Ca} \mathrm{CO}_{3}+18 \mathrm{H}_{2} \mathrm{O} \\
& \mathrm{H}_{2} \mathrm{CO}_{3}+2 \mathrm{~K} \mathrm{OH} \rightarrow \mathrm{K}_{2} \mathrm{CO}_{3}+2 \mathrm{H}_{2} \mathrm{O} \\
& 9 \mathrm{H}_{2} \mathrm{CO}_{3}+9 \mathrm{Ba}(\mathrm{OH})_{2} \rightarrow 9 \mathrm{Ba} \mathrm{CO}_{3}+18 \mathrm{H}_{2} \mathrm{O}
\end{aligned}
$$

Como pode ser visto a água é componente importante nas reações de absorção do dióxido de carbono. Além de facilitar as reações químicas, a água diminui o contato direto entre os agentes anestésicos inalatórios halogenados com os hidróxidos e também com o dióxido de carbono. Quando este contato direto é prolongado e havendo participação de grande número de moléculas (concentrações elevadas de anestésicos) existe maior possibilidade de formação de outros compostos, inclusive o monóxido de carbono ${ }^{10}$.

O homem adulto elimina cerca de 15 litros de óxido de carbono por hora que podem ser absorvidos por $70 \mathrm{~g}$ de cal, então $700 \mathrm{~g}$ de cal podem absorver 10 horas de $\mathrm{CO}_{2}$ expirado e em $1000 \mathrm{~g}$ de cal poderá ser absorvido aproximadamente 14 horas de $\mathrm{CO}_{2}$ expirado.

Sendo as reações do $\mathrm{CO}_{2}$ com a cal reversíveis, parte considerável desta pode ser regenerada.

O período em que a cal é esgotada em um filtro com capacidade de $1000 \mathrm{~g}$ é de pelo menos 14 horas, isto, considerando um sistema fechado com fluxo de gases frescos igual ao consumo de oxigênio. Quando o fluxo de gás fresco é igual ou acima de volume minuto inspiratório a absorção de $\mathrm{CO}_{2}$ pela cal é praticamente nula e em fluxos intermediários a absorção é também intermediária podendo o período de exaustão da cal ser o dobro ou até maior, de acordo com o fluxo.

O indicador mostra a exaustão da cal que deixa de ser branca e passa a ter a cor violeta, quando é usado o etil violeta. Havendo a reação de reversão, a cal volta a cor branca e pode ser usada por "algum" tempo até nova mudança de cor. Este tempo não pode ser precisamente determinado, deve ser observada a mudança da cor e também se está aquecendo que é um sinal da reação exotérmica entre $\mathrm{CO}_{2}$ e a cal.

A reação entre o $\mathrm{CO}_{2}$ e uma base forte como o hidróxido de sódio ou o hidróxido de potássio libera mais calor do que a reação entre o ácido carbônico $\left(\mathrm{H}_{2} \mathrm{CO}_{3}\right)$ que é ácido fraco e as bases fortes, deste modo pode-se compreender a importância de uma certa quantidade de água capaz de fazer a primeira reação com o $\mathrm{CO}_{2}$ e formar o ácido carbônico para prosseguir com a segunda reação com os hidróxidos de cálcio e sódio, eventualmente bário e potássio e formar os carbonatos.

A presença da água na cal tem importância também para evitar a absorção dos anestésicos halogenados para dentro dos grânulos o que poderia retardar a indução anestésica e também promover maior degradação metabólica destes agentes.

O contato dos anestésicos halogenados com a cal pode resultar na metabolização destes agentes. Há relatos desta ocorrência com todos os anestésicos em uso corrente halotano, isoflurano, desflurano e sevoflurano. O que sofre maior 
metabolização é o sevoflurano cujo produto maior de degradação é o composto $A^{11,12}$.

Alguns fatores interferem na absorção e degradação destes agentes e de modo especial do sevoflurano; baixo fluxo de gás fresco, uso da cal baritada, alta concentração do agente, alta temperatura da cal $\left(\leq 70^{\circ} \mathrm{C}\right)$ e desidratação da cal ${ }^{13,14}$.

\section{DEGRADAÇÃO DE ANESTÉSICOS HALOGENADOS PELA CAL SODADA}

A cal sodada degrada os anestésicos inalatórios halogenados através de reações exotérmicas. O Composto A formado pela metabolização do sevoflurano é também um halogenado. Outra reação degrada o sevoflurano transformando em formaldeído ácido hidrofluorídico com formação de metanol a partir do formaldeído. O metanol combinando com o composto A forma o composto B que combinando com outros metabólitos e sendo desflorinado forma o composto $C^{15-17}$. Há relatos de que a absorção e metabolização dos anestésicos halogenados pela cal sodada aumenta quando o conteúdo de água diminui para valores abaixo de 10\%. Da mesma forma a degradação destes agentes pela cal aumenta linearmente com o aumento da temperatura. Isto é bem observado com o sevoflurano ${ }^{17,18}$.

A primeira reação na absorção do $\mathrm{CO}_{2}$ pela cal se processa entre este gás e a água formando o ácido carbônico que a seguir vai reagir com os hidróxidos de cálcio, sódio e potássio ou também de bário. Esta reação é exotérmica, no entanto há mais liberação de calor quando é feita entre o $\mathrm{CO}_{2}$ e os hidróxidos.

O aumento da temperatura aumenta a metabolização dos halogenados e esta metabolização aumenta mais ainda esta degradação metabólica.

Além de produzir produtos tóxicos, o contato dos agentes halogenados com a cal sodada pode resultar na formação de monóxido de carbono ( $\mathrm{CO}$ ). Isto ocorre mais freqüentemente quando alguns destes fatores estão associados: uso de cal baritada, temperatura da cal elevada, cal desidratada, altas concentrações de anestésico e período prolongado da absorção ${ }^{19,20}$.

Níveis mais elevados de CO têm sido descrito com o uso de desflurano nas condições mencionadas. Estas condições tem sido preparadas deliberadamente em estudos experimentais. A literatura não relata casos clínicos sobre esta ocorrência com este agente.

Deve ser observado que a formação de produtos tóxicos como o composto A pode causar sérios danos ao organismo desde a lesão renal e hepática até a encefálica. No caso da liberação do monóxido de carbono, este pode combinar-se com a hemoglobina, impedindo a ligação desta com o oxigênio, prejudicando sensivelmente seu transporte levando o paciente a hipóxia que pode ser grave. A magnitude desta ocorrência clínica vai depender da quantidade de monóxido de carbono formada em um minuto em relação ao consumo de oxigênio do paciente.

A formação em grande escala de monóxido de carbono de $715 \mathrm{ml} \mathrm{em} 2$ horas, corresponde a $6 \mathrm{ml} \cdot \mathrm{min}^{-1}$ que está próxi- ma à metade do consumo de oxigênio do recém-nascido de 3 $\mathrm{kg}$ que é aproximadamente $12 \mathrm{ml} \cdot \mathrm{min}^{-1}$, CO nesta quantidade pelo contato de anestésico com a cal só é obtida experimentalmente.

Deve ser observado que o risco maior de intoxicação pelo CO com uso de halogenados e cal sodada é sempre maior em crianças ou adulto de baixo peso com pequeno consumo de oxigênio.

Wissing ${ }^{21}$, relatou dois casos que ocorreram na Alemanha com uso de sevoflurano ${ }^{22}$. Coincidentemente este agente é um dos que menos forma $\mathrm{CO}$ em contato com a cal sodada como descrito em trabalho experimental desenvolvido com todos os halogenados em uso atualmente ${ }^{21}$.

A intoxicação pelo monóxido de carbono passa facilmente desapercebida porque ocorre muito rapidamente, o CO tem cerca de 200 vezes mais afinidade pela hemoglobina do que o oxigênio. Esta combinação ( $\mathrm{HbCO}$ ) forma um pigmento cor-de-rosa semelhante a oxihemoglobina $\left(\mathrm{HbO}_{2}\right)$, desloca a curva de saturação para esquerda e apresenta repercussões clínicas como taquicardia e aumento do tempo de coagulação do sangue. Estas alterações podem aparecer com baixas taxas de saturação de $\mathrm{HbCO}$, cerca de $4 \%$ a $8 \%{ }^{21,23}$.

Desde o inicio do seu uso, a cal sodada apresentou algumas complicações que resultaram em dificuldade, no entanto as grandes vantagens que oferecia em relação à redução do fluxo de gases frescos, despoluição da sala de cirurgia e umidificação do sistema de inalação fizeram com que continuassem as pesquisas para que pudesse ser melhorada e corrigida de forma que a continuidade da sua utilização fosse assegurada.

A falta de hidróxido de cálcio ou a grande predominância de hidróxido de sódio produzia calor excessivamente, sendo necessário a correção na sua composição que passou a ter mais hidróxido de cálcio e bem menos hidróxido de sódio e também hidróxido de potássio .

Outra dificuldade foi a adição correta da quantidade de água até que ficou estabelecido o valor percentual ideal entre $14 \%$ e $21 \%$.

Atualmente existem duas preocupações básicas em relação a composição da cal com objetivo de evitar a formação de substâncias tóxicas, quando se usa os anestésicos halogenados.

Inicialmente deve-se pensar na maneira correta de se usar a cal suficientemente hidratada.

A presença adequada da água na cal, ou seja, nas quantidades recomendadas, dificulta a absorção do anestésico para o interior do granulo de cal, de forma que quando isto acontece há redução da concentração inspirada do anestésico no momento em que se deseja que esteja mais elevada (indução) e aumenta quando se deseja que esteja baixa (regressão). Além disto, aumenta o contato dos halogenados com a cal facilitando a metabolização destes agentes e a formação de produtos tóxicos.

Outro aspecto importante a considerar na hidratação da cal é que durante a absorção do dióxido de carbono, a primeira reação é entre este gás e a água formando um ácido fraco que é o ácido carbônico, cuja reação com os hidróxidos de bases 
fortes são menos exotérmicas do que a reação direta entre o $\mathrm{CO}_{2}$ e estas bases fortes.

Afalta ou a redução significativa de água que possa atingir níveis considerados críticos, menor do que $4,5 \%$ pode inclusive contribuir de forma importante e determinante para o grande aumento da temperatura na cal resultando em maior metabolização dos anestésicos halogenados e certamente a produção de substâncias potencialmente tóxicas e outras já comprovadamente tóxicas para o organismo humano ${ }^{18}$.

Existe uma reação em cadeia a partir da cal sodada desidratada ou ressecada com baixos volumes percentuais de água. Há aumento da temperatura, maior absorção de anestésico para o interior do grânulo de cal e em seguida maior degradação das moléculas destes agentes e conseqüentemente a produção de composto $\mathrm{A}, \mathrm{CH} 2 \mathrm{~F}-\mathrm{O}-\mathrm{C}(\mathrm{CF} 3)=\mathrm{CF} 2$, pela reação dos hidróxidos com o sevoflurano. O monóxido de carbono, também é produzido pela reação entre os halogenados e as bases da cal ${ }^{18}$. Estes produtos são reconhecidamente nocivos ao homem. O composto Aé nefrotóxico, podendo produzir lesão renal irreversível e o monóxido de carbono leva a hipóxia grave com alterações sobre a coagulação sangüínea ${ }^{24}$.

\section{COMO EVITAR A FORMAÇÃO DE PRODUTOS TÓXICOS}

Além dos cuidados para a hidratação da cal sodada é possível utilizar a cal sem conter o hidróxido de sódio e o hidróxido de potássio que são bases fortes e produzem reações exotérmicas com grande aumento da temperatura ${ }^{25}$.

Estudo experimental demonstrou que usando a cal sodada desidratada sem conter hidróxido de sódio e hidróxido de potássio, contendo somente hidróxido de cálcio a produção de monóxido de carbono é muito menor na presença de desflurano e a produção de composto A é menor na presença de sevoflurano. Com a utilização desta cal contendo somente hidróxido de cálcio e sendo hidratada normalmente, a produção destas substâncias indesejáveis é ainda menor ${ }^{26}$.

Na prática clínica deve-se ter o cuidado para usar a cal sodada mais recente possível e quando esta fica exposta ao meio ambiente por muitas horas, por exemplo, um final de semana (mais de 48 horas) recomenda-se colocar água (de preferência destilada) na relação de $25 \mathrm{ml}$ para cada $500 \mathrm{~g}$ de cal. Estando a indústria de cal sodada informada sobre o assunto deve-se preferir aquela que tenha somente o hidróxido de cálcio e seja desprovida de hidróxido e sódio e hidróxido de potássio.

\section{CONCLUSÃO}

O uso da cal sodada no sistema de inalação do aparelho de anestesia continua sendo desejável pelas grandes vantagens que oferece, redução do fluxo de gás fresco, e do consumo de anestésico, despoluição da sala de cirurgia e manutenção da umidade da via aérea. No entanto é necessário que o anestesiologista tenha alguns cuidados sobre a composição da cal, preferindo aquela que é suficientemente hi- dratada (15\%) e tenha apenas o hidróxido de cálcio como base para absorver o dióxido de carbono.

Em caso de dúvida ou suspeição de formação de produto tóxico deve-se aumentar o fluxo de gases frescos (oxigênio) para um mínimo de 1 volume minuto respiratório e adicionar água destilada à cal sodada, cerca de $25 \mathrm{ml}$ para cada $500 \mathrm{~g}$ de cal.

\section{Preventing Toxic Substances Production during Carbon Dioxide Absorption by Soda Lime with Halogenate Anesthetics}

\author{
Renato Ângelo Saraiva, TSA, M.D.

\section{INTRODUCTION}

Carbon dioxide absorption in the anesthesia machine allows the use of lower fresh gases flow to decrease anesthetic consumption, maintain body temperature, retain airway humidity and prevent operating room pollution.

Wilson ${ }^{1}$ and Waters ${ }^{2}$ have reported that carbon dioxide absorption was first used in anesthesia in 1906 by a German surgeon who has used a filter of underground mines life-saving equipment. According to the same studies, pharmacologist Dennis Jackson has developed in 1915 a soda lime filter based on sodium hydroxide, but carbon dioxide absorption would result in major temperature increase. For this reason, Wilson has modified lime composition which became calcium hydroxide-based.

In 1923, Ralph Waters started using carbon dioxide absorption routinely in anesthesia and has published his results with a $500 \mathrm{~mL}$ filter placed close to patients' mouth in $1926^{3}$. Fresh gases flow was $0.5 \mathrm{~L} . \mathrm{min}^{-1}$ and would enter the upper part of the filter connected to the tracheal tube or facial mask. A respiratory bag was placed in the lower part of the filter. This system was called "to and fro". Currently soda lime composition remains the same: $95 \%$ calcium hydroxide, $4.5 \%$ sodium hydroxide and $0.2 \%$ silica to make lime more consistent and prevent powder formation.

To this mixture $10 \%$ to $22 \%$ water was added (mean $15 \%$ ) and then it was formatted into granules ${ }^{4}$.

Then, another soda lime blend was devised containing $1 \%$ potassium hydroxide, $4 \%$ sodium hydroxide, silica (small amounts, approximately $0.2 \%$ ), $14 \%$ to $19 \%$ water and enough calcium hydroxide to complete $100 \%{ }^{5}$.

Barium lime has $20 \%$ barium hydroxide, $1 \%$ potassium hydroxide, less water than soda lime (approximately $8 \%$ ) and calcium hydroxide to complete $100 \%{ }^{6}$.

In an attempt to make granules more resistant to powder transformation, barium lime was introduced to replace part of calcium hydroxide (approximately $20 \%$ ) by barium hydroxide. The latter, however, produces more heat during carbon dioxide absorption ${ }^{6}$. 
Small amounts of indicators are used, which change color when $\mathrm{pH}$ decreases. The most popular to date is violet ethyl which is transparent but becomes violet when $\mathrm{pH}$ is below $10.3^{7}$.

Carbon dioxide contact with soda lime produces the following reactions:

In a first stage, carbon dioxide $\left(\mathrm{CO}_{2}\right)$ combines with water $\left(\mathrm{H}_{2} \mathrm{O}\right)$ in lime forming carbonic acid $\left(\mathrm{H}_{2} \mathrm{CO}_{3}\right)$.

$\mathrm{CO}_{2}{ }^{+} \mathrm{H}_{2} \mathrm{O} \rightarrow \mathrm{H}_{2} \mathrm{CO}_{3}$

In a second stage, carbonic acid reacts with sodium hydroxide $(\mathrm{OH} \mathrm{Na})$ and calcium hydroxide $\mathrm{Ca}(\mathrm{OH})_{2}$ producing sodium carbonate $\left(\mathrm{CO}_{3} \mathrm{Na}_{2}\right)$ and calcium carbonate $\left(\mathrm{CO}_{3} \mathrm{Ca}\right)$ and releasing water.

$2 \mathrm{NaOH}+2 \mathrm{H}_{2} \mathrm{CO}_{3}+\mathrm{Ca}(\mathrm{OH})_{2} \rightarrow \mathrm{CaCO}_{3}+\mathrm{Na}_{2} \mathrm{Co}_{3}+4 \mathrm{H}_{2} \mathrm{O}$

Carbon dioxide reactions with barium lime are as follows ${ }^{8,9}$ :

$9 \mathrm{CO}_{2}+9 \mathrm{H}_{2} \mathrm{O} \rightarrow 9 \mathrm{H}_{2} \mathrm{CO}_{3}$

$9 \mathrm{H}_{2} \mathrm{CO}_{3}+9 \mathrm{Ca}(\mathrm{OH})_{2} \rightarrow 9 \mathrm{Ca} \mathrm{CO}_{3}+18 \mathrm{H}_{2} \mathrm{O}$

$\mathrm{H}_{2} \mathrm{CO}_{3}+2 \mathrm{~K} \mathrm{OH} \rightarrow \mathrm{K}_{2} \mathrm{CO}_{3}+2 \mathrm{H}_{2} \mathrm{O}$

$9 \mathrm{H}_{2} \mathrm{CO}_{3}+9 \mathrm{Ba}(\mathrm{OH})_{2} \rightarrow 9 \mathrm{Ba} \mathrm{CO}+18 \mathrm{H}_{2} \mathrm{O}$

As it can be seen, water is a major component in carbon dioxide absorption reactions. In addition to helping chemical reactions, water decreases direct contact of halogenate inhalational anesthetics with hydroxides and carbon dioxide. When there is direct prolonged contact with the participation of a large number of molecules (high anesthetic concentrations), there is a higher probability for the formation of other compounds, including carbon monoxide ${ }^{10}$.

Adults eliminate approximately $15 \mathrm{~L}$ of carbon dioxide per hour, which may be absorbed by $70 \mathrm{~g}$ lime, so $700 \mathrm{~g}$ lime may absorb 10 hours of expired $\mathrm{CO}_{2}$, and $1000 \mathrm{~g}$ lime may absorb approximately 14 hours of expired $\mathrm{CO}_{2}$.

Since $\mathrm{CO}_{2}$ reactions with lime are reversible, considerable part of it may be regenerated. The period in which lime is depleted in a $1000 \mathrm{~g}$ filter is at least 14 hours, considering a close system with fresh gases flow equal to oxygen consumption. When fresh gases flow is equal to or above inspiratory minute volume, absorption is almost null, in intermediary flows the absorption is also intermediary and lime depletion period may be twice or even higher, depending on the flow.

The indicator shows lime depletion turning from white to violet when violet ethyl is used. With reversion reaction, lime returns to white and may be used for "some" time before another color change. This time is not precise. Color change should be observed in addition to heating which is an sign of exothermal reaction between $\mathrm{CO}_{2}$ and lime.
$\mathrm{CO}_{2}$ reaction with a strong base such as sodium or potassium hydroxide releases more heat than the reaction of carbonic acid $\left(\mathrm{H}_{2} \mathrm{CO}_{3}\right)$, which is a weak acid, and strong bases. So it is easy to understand the importance of a certain amount of water to complete the first reaction with $\mathrm{CO}_{2}$ and form carbonic acid to continue with the second reaction with calcium and sodium hydroxides, eventually barium and potassium, and form carbonates.

The presence of water in lime is also important to prevent halogenate anesthetic absorption by granules which could delay anesthetic induction and promote further metabolic degradation of those agents.

The contact of halogenate agents with lime may result in their metabolism. There are reports of this event with all anesthetics currently used: halothane, isoflurane, desflurane and sevoflurane. Sevoflurane has the highest metabolic rate and its major degradation product is Compound $A^{11,12}$.

Some factors interfere with absorption and degradation of those agents, especially sevoflurane: low fresh gases flow, barium lime, high agent concentration, high lime temperature $\left(\leq 70^{\circ} \mathrm{C}\right)$ and lime dehydration ${ }^{13,14}$.

\section{HALOGENATE ANESTHETICS DEGRADATION BY SODA LIME}

Soda lime degrades inhalational halogenate anesthetics through exothermal reactions. Compound $A$, formed by sevoflurane metabolism is also halogenate. A different reaction degrades sevoflurane transforming it into formaldehyde hydrofluoric acid with methanol formation as from formaldehyde. Methanol combined with compound Aforms compound $B$, which combined with other metabolites and being defluorinated forms compound $\mathrm{C}^{15-17}$.

There are reports that halogenate anesthetics absorption and metabolism by soda lime increase when water content decreases to below $10 \%$. Similarly, these agents' degradation by lime is linearly increased with increased temperature. This is clearly seen with sevoflurane ${ }^{17,18}$

The first reaction when $\mathrm{CO}_{2}$ is absorbed by lime is processed between this gas and water generating carbonic acid which then reacts with calcium, sodium, potassium and barium hydroxides. This is an exothermal reaction but there is more heat release when the reaction is between $\mathrm{CO}_{2}$ and hydroxides.

Increased temperature increases halogenate metabolism and such metabolism further increases this metabolic degradation.

In addition to producing toxic substances, the contact of halogenate agents with soda lime may result in carbon monoxide (CO). This is more frequent when some of the following factors are associated: barium lime, high lime temperature, dehydrated lime, high anesthetic concentrations and prolonged absorption time ${ }^{19,20}$.

Higher CO levels have been described with desflurane in the previous-mentioned conditions. These conditions have been 
deliberately prepared in experimental studies. There are no clinical cases in the literature about this event.

It should be observed that formation of toxic substances such as compound A may promote severe damage to kidney, liver and brain. Carbon monoxide may combine with hemoglobin, preventing its binding to oxygen and severely impairing its transportation, leading patients to hypoxia which may be severe. The magnitude of this clinical event will depend on the amount of carbon monoxide formed in one minute as compared to oxygen consumption.

High carbon monoxide volumes of $715 \mathrm{~mL}$ in two hours, correspond to $6 \mathrm{~mL}$. $\mathrm{min}^{-1}$, which is close to half oxygen consumption of a 3-kg neonate, which is approximately $12 \mathrm{~mL} \cdot \mathrm{min}^{-1}$. This amount of $\mathrm{CO}$ by contact of anesthetics with lime is only experimentally obtained.

It should be noted that $\mathrm{CO}$ intoxication risk with halogenate and soda lime is always higher in children or low weight adults with low oxygen consumption. Wissing ${ }^{21}$ has reported two cases in Germany with sevoflurane ${ }^{22}$. Coincidentally, this is the agent less forming $\mathrm{CO}$ in contact with soda lime, as described in an experimental study with all currently used halogenates ${ }^{21}$.

Carbon monoxide intoxication goes easily unnoticeable for being very fast. CO has approximately 200 times more affinity to hemoglobin as compared to oxygen. This combination $(\mathrm{HbCO})$ forms a pink pigment similar to oxyhemoglobin $\left(\mathrm{HbO}_{2}\right)$, shifts saturation curve to the left and promotes clinical repercussions such as tachycardia and increased coagulation time. These changes may be seen with low $\mathrm{HbCO}$ saturation rates, approximately $4 \%$ to $8 \%{ }^{21,23}$.

Since the beginning, soda lime has presented some complications which have resulted in its difficult use, however major advantages, such of decreasing fresh gases flow and operating room pollution, and improved inhalation system humidity, have pushed research forward to improve it and assure the continuity of its use.

Lack of calcium hydroxide or major predominance of sodium hydroxide would produce excessive heat being necessary an adjustment on its composition which started to have more calcium hydroxide and much less sodium and potassium hydroxide.

Another difficulty was the accurate addition of water until the optimal percentage between $14 \%$ and $21 \%$ was established.

There are currently two basic concerns with lime composition to prevent toxic substances formation when it is used with halogenate anesthetics.

First one should think about the correct way to use sufficiently hydrated lime.

Adequate amount of water on lime, that is, in the recommended amounts, impairs anesthetic absorption by lime granules. If this occurs there is decreasing anesthetic inspired concentration when it should be high (induction) and increasing it when it should be low (emergence). In addition, it increases contact of halogenates with lime, favoring their metabolism and the formation of toxic substances.

Another important aspect of lime hydration is that during carbon dioxide absorption, the first reaction is between this gas and water forming carbonic acid which is a weak acid. The reaction of this acid with strong base hydroxides is less exothermal as compared to the direct reaction of $\mathrm{CO}_{2}$ with such strong bases.

Lack or significant water decrease reaching critical levels below $4.5 \%$ may even contribute in a major and determinant way to high lime temperature increase resulting in more halogenate anesthetic metabolism and surely the production of potentially or knowingly toxic substances ${ }^{18}$.

There is a chain reaction as from dehydrated or dried out soda lime. There is temperature increase, more anesthetic absorption by lime granules and more degradation of these agents' molecules with the production of compound $\mathrm{A}, \mathrm{CH} 2 \mathrm{~F}-\mathrm{O}-\mathrm{C}$ $(C F 3)=C F 2$, by reaction of hydroxides with sevoflurane. Carbon monoxide is also produced by the reaction of halogenates and lime bases ${ }^{18}$. These products are knowingly noxious for humans. Compound $A$ is nephrotoxic and may produce irreversible renal injury, and carbon monoxide leads to severe hypoxia with blood coagulation changes ${ }^{24}$.

\section{PREVENTING TOXIC SUBSTANCES PRODUCTION}

In addition to care with soda lime hydration, it is possible to use lime without sodium and potassium hydroxides which are strong bases and produce exothermal reaction with major temperature increase ${ }^{25}$.

An experimental study has shown that with dehydrated soda lime without sodium and potassium hydroxide, just with calcium hydroxide, carbon monoxide production is much lower in the presence of desflurane, and compound A production is lower in the presence of sevoflurane. With this same lime, however adequately hydrated, the production of those undesirable substances is even lower ${ }^{26}$.

Care should be taken to use the newest possible soda lime and when it is exposed to environment for many hours, such as during weekends (more than 48 hours) it is recommended to add water (preferably distilled water) in the ratio of $25 \mathrm{~mL}$ for each $500 \mathrm{~g}$ of lime.

Sodium and potassium hydroxide-free soda lime, with calcium hydroxide only, should be preferred.

\section{CONCLUSION}

Soda lime in the anesthesia machine inhalation system is still desirable for its major advantages, such as decreasing fresh gases flow, anesthetic consumption and operating room pollution and maintaining airway humidity. However it is necessary that anesthesiologists take some care with lime composition, preferring those sufficiently hydrated (15\%) and with just calcium hydroxide as base to absorb carbon dioxide. In case of doubt or suspicion of toxic substance formation fresh gases flow (oxygen) should be increased to a minimum of 1 respiratory minute/volume and distilled water should be added to soda lime, approximately $25 \mathrm{~mL}$ for each $500 \mathrm{~g}$ of lime. 


\section{REFERÊNCIAS - REFERENCES}

01. Wilson RE - Sodalime absorbent for industrial purposes. Ind Eng Chem, 1920;12:1000-1006.

02. Waters RM - Clinical scope and utility of carbon dioxide filtration with inhalation anesthesia. Anesth Analg, 1924;3:20-28.

03. Waters RM - Advantages and techniques of carbon dioxide filtration with inhalation anesthesia. Anesth Analg, 1926;5:160-166.

04. Adriani $\mathrm{J}$ - The Effect of the varying of moisture content of soda lime upon the efficiency of carbon dioxide absorption. Anesthesiology, 1945;6:163-171.

05. Adriani J - Disposal of carbon dioxide from devices used for inhalation anesthesia. Anesthesiology, 1944;6:35-52.

06. Adriani J, Batten DH - The efficiency of moisture of barium and calcium hydroxides in the absorption of $\mathrm{CO}_{2}$ rebreathing appliances. Anesthesiology, 1945;6:35-52.

07 . Adriani J - Soda lime containing indicators. Anesthesiology, 1944;5:45-53.

08. Hale DE - The rise and fall of soda lime. Anesth Analg, 1967;46: 648-655.

09. Adriani J - Rebreathing in anesthesia. South Med J, 1942;35: 798-804.

10. Grodin WK, Epstein MA, Epstein RA - Mechanisms of halothane adsorption by dry soda-lime. Br J Anaesth, 1982;54:561-565.

11. Strum DP, Jonson BH, Eger II EI - Stability of sevoflurane in soda lime. Anesthesiology, 1987;67:779-781

12. Lin J, Laster MJ, Eger II El et al - Absorption and degradation of sevoflurane and isoflurane in a conventional anesthetic circuit. Anesth Analg, 1991;72:785-789.

13. Frink Jr EJ, Malan TP, Morgan SE et al - Quantification of the degradation products of sevoflurane in two $\mathrm{CO}_{2}$ absorbants during low-flow anesthesia in surgical patients. Anesthesiology, 1992;77:1064-1069.

14. Fang ZX, Eger II EI - Factors affecting the concentration of compound $A$ resulting from the degradation of sevoflurane by soda lime and baralyme in a standard anesthetic circuit. Anesth Analg, 1995;81:564-568.

15. Hanaki C, Fuji K, Morio M et al - Decomposition of sevoflurane by sodalime. Hiroshima J Med Sci, 1987;36:61-67.

16. Brown ES, Bakamjian V, Seniff AM - Performance of absorbents: effect of moisture. Anesthesiology, 1959;20:613-617

17. Eger II EI, Strum DP - The absorption and degradation of isoflurane and I-653 by dry soda lime at various temperatures. Anesth Analg, 1987;66:1312-1315.

18. Strum DP, Eger II EI - The degradation, absorption and solubility of volatile anesthetics in soda lime depend on water content. Anesth Analg, 1994;78:340-348.

19. Fang ZX, Eger II El, Laster MJ et al - Carbon monoxide production from degradation of desflurane, enflurane, isoflurane, halothane, and sevoflurane by soda lime and baralyme. Anesth Analg, 1995:80:1187-1193.

20. Harrison N, Knowles AC, Welchew EA - Carbon monoxide within circle systems. Anaesthesia, 1996;51:1037-1040.

21. Wissing $\mathrm{H}$, Kuhn I, Warnken $\mathrm{U}$ et al - Carbon monoxide production from desflurane, enflurane, halothane, isoflurane, and sevoflurane with dry soda lime. Anesthesiology, 2001;95: 1205-1212.

22. Braum J, Sitte T, Straub JM et al - Die reaction on sevoflurane mettrickenem atemkalk. Uberlegungen anlablich eines aktuellen zwischen falls. Anasth Intesivmed, 1998;39:11-16.

23. Narkool DM, Kirkpaltric JN - Treatment of acute carbon monoxide poising with hyperbaric oxygen. A review of 115 cases. Ann Emerg Med, 1985;14:1168-1182.
24. Frink Jr EJ, Nogami WM, Morgan SE et al - High carboxyhemoglobin concentrations occur in swine during desflurane anesthesia in presence of partially dried carbon dioxide absorbents. Anesthesiology, 1997;87:308-316.

25. Goldberg ME, Cantillo J, Gratz I et al - Dose of compound A, not sevoflurane, determines changes in the biochemical markers of renal injury in healthy volunteers. Anesth Analg, 1999;88: 437-445.

26. Neumann MA, Laster MJ, Weiskopf RB et al - The elimination of sodium and potassium hydroxides from desiccated soda lime diminishes degradation of desflurane do carbon monoxide and sevoflurane to compound A but does not compromise carbon dioxide absorption. Anesth Analg, 1999;89:768-773.

\section{RESUMEN}

Saraiva RA - Como Evitar la Formación de Substancias Tóxicas durante la Absorción de Dióxido de Carbono por la Cal Sodada con Uso de Anestésicos Halogenados

JUSTIFICATIVA Y OBJETIVOS: La cal sodada desde el inicio de su uso siempre presentó algunas complicaciones que resultaron en dificultad en su aplicabilidad. No entanto, debido a las grandes ventajas que ofrecía en relación a la reducción del flujo de gases frescos, despolución de la sala de cirugía y humidificación del sistema de inhalación y vía aérea, hicieron con que continuasen las pesquisas para que pudiese ser mejorada y corregida de forma que la continuidad de su utilización sea asegurada. Actualmente existe el problema de la deshidratación con elevación de la temperatura y de la degradación metabólica de los anestésicos halogenados que necesitan de cuidados especiales para evitar la formación de productos tóxicos.

CONTENIDO: Existe una reacción en cadena a partir de la cal sodada deshidratada o resecada con bajos volúmenes porcentuales de agua. Hay aumento de la temperatura, mayor absorción de anestésico halogenado para el interior del granulo de cal y en seguida mayor degradación metabólica de las moléculas de estos agentes y consecuentemente la producción de substancias tóxicas como el Compuesto A por la reacción de los hidróxidos con el sevoflurano. Hay también formación de monóxido de carbono producido de la misma forma por la reacción entre los halogenados y las bases fuertes de la cal. El compuesto $A$ es nefrotóxico y el monóxido de carbono lleva a la hipóxia y alteraciones graves de la coagulación de la sangre. Además de los cuidados para la hidratación de la cal sodada es posible usar ésta sin contener las bases fuertes como los hidróxidos de potasio y de sodio, conteniendo apenas hidróxido de calcio para evitar excesivo aumento de la temperatura y grande degradación metabólica de los halogenados sin perjudicar la absorción del dióxido de carbono.

CONCLUSIONES: Se debe tener el cuidado en usar la cal sodada lo antes posible y cuando ella queda expuesta al medio ambiente (aire seco) por muchas horas como por ejemplo en un final de semana (mas de 48 horas) es recomendable colocar agua, de preferencia destilada, en la relación de $25 \mathrm{ml}$ para cada $500 \mathrm{~g}$ de cal. Actualmente la industria está bien informada sobre el problema de la composición de la cal, entonces, se debe preferir la cal sodada que tenga solamente el hidróxido de calcio y sea totalmente desprovista de hidróxido de potasio e hidróxido de sodio. 